\title{
RESEARCH
}

Open Access

\section{Cooking skills related to potential benefits for dietary behaviors and weight status among older Japanese men and women: a cross-sectional study from the JAGES}

\author{
Yukako Tani ${ }^{*^{*}}$, Takeo Fujiwara ${ }^{1}$ and Katsunori Kondo ${ }^{2,3}$
}

\begin{abstract}
Background: Poor cooking skills have been linked to unhealthy diets. However, limited research has examined associations of cooking skills with older adults' health outcomes. We examined whether cooking skills were associated with dietary behaviors and body weight among older people in Japan.

Methods: We used cross-sectional data from the 2016 Japan Gerontological Evaluation Study, a self-report, population-based questionnaire study of men $(n=9143)$ and women $(n=10,595)$ aged $\geq 65$ years. The cooking skills scale, which comprises seven items with good reliability, was modified for use in Japan. We calculated adjusted relative risk ratios of unhealthy dietary behaviors (low frequency of home cooking, vegetable/fruit intake; high frequency of eating outside the home) using logistic or Poisson regression, and relative risk ratios of obesity and underweight using multinomial logistic regression.

Results: Women had higher levels of cooking skills, compared with men. Women with a moderate to low level of cooking skills were 3.35 (95\% confidence interval [CI]: 2.87-3.92) times more likely to have a lower frequency of home cooking and 1.61 (95\% Cl: 1.36-1.91) times more likely to have a lower frequency of vegetable/fruit intake, compared with women with a high level of cooking skills. Men with a low level of cooking skills were 2.56 (95\% Cl: 2.36-2.77) times more likely to have a lower frequency of home cooking and 1.43 (95\% Cl: 1.06-1.92) times more likely to be underweight, compared with men with a high level of cooking skills. Among men in charge of meals, those with a low level of cooking skills were 7.85 (95\% Cl: 6.04-10.21) times more likely to have a lower frequency of home cooking, 2.28 ( $95 \%$ Cl: 1.36-3.82) times more likely to have a higher frequency of eating outside the home, and 2.79 (95\% Cl: 1.45-5.36) times more likely to be underweight, compared with men with a high level of cooking skills. Cooking skills were unassociated with obesity.

Conclusions: A low level of cooking skills was associated with unhealthy dietary behaviors and underweight, especially among men in charge of meals. Research on improving cooking skills among older adults is needed.
\end{abstract}

Keywords: Cooking skill, Home cooking, Eating out, Vegetable and fruit intake, Obesity, Underweight

\footnotetext{
* Correspondence: tani.hlth@tmd.ac.jp

'Department of Global Health Promotion, Tokyo Medical and Dental University (TMDU), 1-5-45, Yushima, Bunkyo-ku, Tokyo 113-8519, Japan

Full list of author information is available at the end of the article
}

(c) The Author(s). 2020 Open Access This article is licensed under a Creative Commons Attribution 4.0 International License, which permits use, sharing, adaptation, distribution and reproduction in any medium or format, as long as you give appropriate credit to the original author(s) and the source, provide a link to the Creative Commons licence, and indicate if changes were made. The images or other third party material in this article are included in the article's Creative Commons licence, unless indicated otherwise in a credit line to the material. If material is not included in the article's Creative Commons licence and your intended use is not permitted by statutory regulation or exceeds the permitted use, you will need to obtain permission directly from the copyright holder. To view a copy of this licence, visit http://creativecommons.org/licenses/by/4.0/ The Creative Commons Public Domain Dedication waiver (http://creativecommons.org/publicdomain/zero/1.0/) applies to the data made available in this article, unless otherwise stated in a credit line to the data. 


\section{Background}

There are increasing calls to return to home cooking to prevent poor diets and chronic diet-related diseases [1]. A systematic review has reported the dietary benefits of eating home-cooked meals, including greater consumption of fruits and vegetables, enhanced nutrient intake, and higher diet quality [2]. A recent cross-sectional study showed that eating home-cooked dinners was associated with greater dietary guideline compliance, without significantly increasing food expenditures [3]. Although studies related to the effects of home cooking on health outcomes are limited, a recent large population-based study in the United Kingdom showed that more frequent consumption of home-cooked meals was associated with a greater likelihood of having normal weight and body fat status [4]. Furthermore, a cohort study targeting older people in Taiwan demonstrated that older adults who cooked more than five times per week had approximately $40 \%$ lower risk of death, compared with those who did not cook [5]. The study also showed a dose-response relationship, meaning that the risk of death decreased as the frequency of home cooking increased. Despite the benefits of home cooking, the consumption of home-cooked meals has declined and the consumption of out-of-home foods, such as fast food and convenience food, has increased in recent decades in developed countries $[6,7]$.

Cooking skills are one important modifiable factor that can encourage people to cook [2]. In addition to increasing the frequency of home cooking, strengthening people's cooking skills can improve their diet quality. For example, cross-sectional studies have shown an association between high levels of cooking skills and lower consumption of ready meals, convenience food, and ultra-processed food among adults [8-10]. Intervention studies have also shown improving cooking skills to increase cooking confidence and consumption of vegetables and fruits [11, 12]. Most existing studies have focused on dietary benefits among adults, and limited work has examined the associations between cooking skills and health outcomes among older adults.

Population aging is increasing dramatically, and the percentage of the world's population aged over 60 years is projected to nearly double from $12 \%$ in 2015 to $22 \%$ in 2050 [13]. Overall, older adults do not meet the recommendations for a healthy diet [14]. Physiological, social, economic, and psychological factors affect older people's food choices. Physiological factors, such as age-related decline in taste and smell, can lead to decreased appetite and poor dietary habits [15]. Social factors, including lower social engagement and living alone, are also associated with poor diet quality $[15,16]$. Economic factors such as low income and retirement can negate older people's ability to meet their nutritional needs $[17,18]$. Finally, psychological factors including wellbeing and depression are also associated with eating behaviors [16]. However, although cooking skills are a fundamental factor in preparing meals, the effects of cooking skills on dietary behaviors do not seem to have been evaluated among older adults. A systematic review demonstrated associations between culinary interventions and improved dietary factors, including attitudes, self-efficacy, and healthy dietary intake among adults [19]. Focusing on cooking skills as a modifiable factor among older adults is an innovative approach.

The rationales described above indicate that having sufficient cooking skills may be important for healthy aging. More older adults live alone, compared with other age groups: In 43 developing countries, only $1.6 \%$ of people were found to live alone overall, compared with $8.8 \%$ of older adults [20]. In Japan, most older people live alone or with their spouses [21]. Thus, older adults are faced with the task of preparing own meals. Because of changes in living arrangements or spouses becoming unable to cook, the person responsible for cooking at home may change in older age. For example, a widowed man may be in charge of cooking for the first time. Because it is mainly women who are in charge of preparing meals, men have been found to be less confident in their cooking and to have lower levels of cooking skills $[9,22]$. Therefore, men, especially widowed men or men whose spouses are unable to cook, may be at risk of diet-related problems because of their poor cooking skills. To our knowledge, no study has examined the associations between cooking skills and health outcomes among older people who are in charge of meals.

One reason for the limited evidence relating to cooking skills may be the difficulty of assessing cooking skills. Cooking skills have been defined as a set of mechanical or physical skills used in meal preparation, such as chopping, mixing, and heating basic ingredients, as well as conceptual skills related to understanding how food will react when cooked [23]. In addition to the various aspects of cooking skills, the cooking skills required vary depending on culture: For example, cooking methods (e.g., grilling, steaming, stewing, and stir-frying) differ by culture. Several methods have been used to measure cooking skills, but there are few validated and reliable measures of cooking skills [23]. Hartmann et al. conducted a test-retest analysis and designed a reliable cooking skills scale comprising seven items related to the ability to prepare different foods that is applicable to most European cultures [9]. Because this scale rates the ability to prepare general food groups (e.g., bread), it is more versatile than scales that rate the ability to prepare specific meals (e.g., spaghetti Bolognese). Therefore, for the present study, we modified this scale for application in a Japanese population. The first aim of our study was to assess the reliability of this scale in a large-scale 
Japanese population-based study. The second aim was to examine the associations of cooking skills with the frequency of home cooking, the frequency of eating outside the home, the frequency of vegetable/fruit consumption, and body weight status.

\section{Methods}

\section{Study design and participants}

The Japan Gerontological Evaluation Study (JAGES), a large nation-wide research project on aging, was established in 2010 to evaluate the social determinants of healthy aging among older people in Japan $[24,25]$. We used data from the 2016 JAGES, which covered $39 \mathrm{mu}$ nicipalities across Japan and was administered to community-dwelling older adults who were physically and cognitively independent (i.e., without functional disabilities, defined as not being certified as eligible to receive long-term public care insurance system services [26]). From October 2016 to January 2017, self-report questionnaires were mailed to 279,661 older adults aged $\geq 65$ years. The survey was conducted using random sampling in 22 large municipalities and was administered to all eligible residents in 17 small municipalities. A total of 196,438 participants returned the questionnaire (response rate: $70.2 \%)$. In some municipalities, recipients receiving long-term public care insurance benefits were included in the survey by request of the local government, so the target sample was 180,021 older adults, after excluding those who received these benefits. Oneeighth of the sample $(N=22,219)$ were randomly selected to receive a survey module on cooking skills. The present analysis was carried out using data for 19,738 participants (9143 men and 10,595 women), after the following exclusions: participants with missing information on gender $(N=2)$; participants who did not complete the questions related to height and weight $(N=660)$ or dietary behaviors (frequency of home cooking, eating outside the home, and vegetable/fruit intake) $(N=1475)$; participants with missing data on the cooking skills scale $(N=145)$; and participants who were included in this study accidentally who reported limitations in activities of daily living $(N=199)$ to ensure that the sample was actually physically and cognitively independent. Limitations in activities of daily living were assessed with the Independence in Activities of Daily Living index [27] using the following questionnaire item: "Do you need any nursing care or assistance from someone in your daily life?" We excluded participants who answered "I need and receive nursing care or assistance." Participants were informed that participation in the study was voluntary and that completing and returning the questionnaire indicated their consent to participate in the study. The JAGES protocol was approved by the Ethics Committee in Research of Human Subjects at the
National Center for Geriatrics and Gerontology (No. 992) and Chiba University Faculty of Medicine (No. 2493).

\section{Body weight status and dietary behaviors}

Participants reported their height in centimeters and weight in kilograms. Body mass index (BMI) was calculated as weight divided by the square of height $\left(\mathrm{kg} / \mathrm{m}^{2}\right)$. We defined underweight as having a $\mathrm{BMI}<18.5 \mathrm{~kg} / \mathrm{m}^{2}$ and obesity as having a BMI $\geq 27.5 \mathrm{~kg} / \mathrm{m}^{2}$, following the suggested cutoff points for Asians [28]. The evaluated dietary behaviors were the frequency of home cooking, eating outside the home, and vegetable/fruit intake. The frequency of home cooking was assessed using the question "How often do you cook by yourself? Do not include ready-to-eat food" (responses: more than five times a week, three to five times a week, one to two times a week, less than once a week, and never). Respondents who cooked less than two times a week were categorized as having a low cooking frequency for women because more than three times a week has been shown to predict survival among older women [5]. For men, respondents who never cooked were categorized as having a low cooking frequency because more than half of the men indicated that they never cooked (Table 1). The frequency of eating outside the home was assessed using the question "How often do you eat outside the home?" The responses for this item were the same as those for the frequency of home cooking. Respondents who ate outside the home more than three times a week were categorized as having a high frequency of eating outside the home because eating outside the home more than three times a week has been shown to be related to higher BMI and lower serum concentrations of nutrients [29]. The frequency of vegetable and fruit intake was assessed using the question "How often did you eat vegetables and fruits over the past month?" (responses: not at all, less than once a week, once a week, two to three times a week, four to six times a week, once a day, and at least twice a day) [30,31]. Respondents who ate vegetables and fruits less than once a day were categorized as having a low frequency of vegetable and fruit intake. This cutoff point was defined by prevalence to be under $25 \%$ of subjects included (Table 1) because being in the lowest quartile for vegetable and fruit intake has been shown to be associated with poor health outcomes [32-34].

\section{Cooking skills}

As mentioned above, based on the cooking skills scale for European cultural regions [9], we adapted Hartmann's a cooking skills scale for use in Japanese populations. In Japan, a typical meal-known as ichi-ju sansai-consists of a staple food (such as rice), a soup (usually miso), and three dishes (one main dish and two side 
Table 1 Characteristics of older Japanese men and women by level of cooking skills $(n=19,378)$

\begin{tabular}{|c|c|c|c|c|c|c|c|c|c|c|c|}
\hline & \multicolumn{6}{|c|}{ Males $(n=9143)$} & \multicolumn{5}{|c|}{ Females $(n=10,595)$} \\
\hline & \multicolumn{2}{|l|}{$\overline{\text { Total }}$} & \multicolumn{4}{|c|}{ Cooking skill } & \multicolumn{2}{|l|}{ Total } & \multicolumn{3}{|c|}{ Cooking skill } \\
\hline & $n$ & $\%$ & High & Middle & Low & $p$-value & $n$ & $\%$ & High & Middle/Low & $p$-value \\
\hline & & & $\%$ & $\%$ & $\%$ & & & & $\%$ & $\%$ & \\
\hline \multicolumn{12}{|l|}{ Cooking skill } \\
\hline High & 4751 & 52.0 & 100 & 0 & 0 & & 9968 & 94.1 & 100.0 & 0 & \\
\hline Middle & 3269 & 35.8 & 0 & 100 & 0 & & 498 & 4.7 & 0 & 79.4 & \\
\hline Low & 1123 & 12.3 & 0 & 0 & 100 & & 129 & 1.2 & 0 & 20.6 & \\
\hline \multicolumn{12}{|l|}{ Age (years) } \\
\hline $65-69$ & 2855 & 31.2 & 32.7 & 29.8 & 29.2 & $<0.001$ & 3269 & 30.9 & 31.7 & 18.0 & $<0.001$ \\
\hline $70-74$ & 2510 & 27.5 & 26.9 & 29.1 & 25.1 & & 2893 & 27.3 & 27.8 & 19.9 & \\
\hline $75-79$ & 2059 & 22.5 & 22.9 & 22.3 & 21.4 & & 2393 & 22.6 & 22.8 & 19.8 & \\
\hline$\geq 80$ & 1719 & 18.8 & 17.5 & 18.8 & 24.3 & & 2040 & 19.3 & 17.8 & 42.3 & \\
\hline \multicolumn{12}{|l|}{ Education (years) } \\
\hline $\operatorname{Low}(\leq 9)$ & 2626 & 28.7 & 28.2 & 28.1 & 32.7 & 0.002 & 3614 & 34.1 & 33.3 & 46.4 & $<0.001$ \\
\hline Middle (10-12) & 3461 & 37.9 & 36.8 & 39.4 & 37.9 & & 4638 & 43.8 & 44.2 & 37.0 & \\
\hline High ( $\geq 13)$ & 2981 & 32.6 & 34.1 & 31.8 & 28.8 & & 2198 & 20.7 & 21.1 & 14.7 & \\
\hline Other/Missing & 75 & 0.8 & 0.9 & 0.8 & 0.6 & & 145 & 1.4 & 1.3 & 1.9 & \\
\hline \multicolumn{12}{|l|}{ Annual income (million yen) } \\
\hline Low $(<2.00)$ & 3485 & 38.1 & 37.5 & 38.6 & 39.4 & 0.215 & 4110 & 38.8 & 38.5 & 43.1 & $<0.001$ \\
\hline Middle (2.00-3.99) & 3279 & 35.9 & 35.9 & 36.6 & 33.3 & & 3049 & 28.8 & 29.3 & 21.1 & \\
\hline High $(\geq 4.00)$ & 924 & 10.1 & 10.6 & 9.3 & 10.6 & & 885 & 8.4 & 8.5 & 5.9 & \\
\hline Missing & 1455 & 15.9 & 16 & 15.4 & 16.7 & & 2551 & 24.1 & 23.7 & 30.0 & \\
\hline \multicolumn{12}{|l|}{ Marital status } \\
\hline Married & 7788 & 85.2 & 81.6 & 88.0 & 92.0 & $<0.001$ & 6497 & 61.3 & 62.4 & 44.7 & $<0.001$ \\
\hline Widowed & 631 & 6.9 & 8.5 & 5.4 & 4.3 & & 3097 & 29.2 & 28.3 & 43.9 & \\
\hline Divorced & 321 & 3.5 & 4.7 & 2.6 & 1.2 & & 513 & 4.8 & 4.8 & 5.1 & \\
\hline Single & 257 & 2.8 & 3.4 & 2.6 & 1.0 & & 307 & 2.9 & 2.8 & 4.1 & \\
\hline Other/Missing & 146 & 1.6 & 1.8 & 1.3 & 1.5 & & 181 & 1.7 & 1.7 & 2.2 & \\
\hline \multicolumn{12}{|l|}{ Under medical treatment } \\
\hline Cancer (Yes) & 450 & 4.9 & 5.1 & 4.5 & 5.7 & 0.53 & 355 & 3.4 & 3.3 & 4.3 & 0.35 \\
\hline Heart disease (Yes) & 1219 & 13.3 & 13 & 13.6 & 14.2 & 0.84 & 713 & 6.7 & 6.5 & 9.7 & 0.008 \\
\hline Stroke (Yes) & 385 & 4.2 & 3.5 & 4.5 & 6.5 & $<0.001$ & 184 & 1.7 & 1.6 & 4.1 & $<0.001$ \\
\hline Diabetes mellitus (Yes) & 1484 & 16.2 & 16.6 & 15.3 & 17.4 & 0.47 & 1010 & 9.5 & 9.5 & 10.4 & 0.69 \\
\hline Hypertension (Yes) & 3913 & 42.8 & 42.1 & 43.5 & 43.8 & 0.66 & 4391 & 41.4 & 41.0 & 48.2 & 0.002 \\
\hline Hyperlipidemia (Yes) & 933 & 10.2 & 10.3 & 10.4 & 9.4 & 0.92 & 1658 & 15.6 & 15.8 & 13.2 & 0.19 \\
\hline \multicolumn{12}{|l|}{ Main way of preparing meals } \\
\hline Cook by yourself & 1283 & 14.0 & 22.2 & 6.1 & 2.2 & $<0.001$ & 8714 & 82.2 & 84.0 & 54.9 & $<0.001$ \\
\hline Family member cook & 6785 & 74.2 & 65.1 & 82.5 & 88.5 & & 977 & 9.2 & 7.9 & 30.3 & \\
\hline Buy cooked meals & 307 & 3.4 & 2.8 & 4.3 & 2.8 & & 132 & 1.2 & 1.1 & 4.1 & \\
\hline Use home-delivery services & 81 & 0.9 & 0.9 & 0.7 & 1.4 & & 46 & 0.4 & 0.4 & 1.0 & \\
\hline Other/Missing & 687 & 7.5 & 8.9 & 6.3 & 5.1 & & 726 & 6.9 & 6.7 & 9.7 & \\
\hline \multicolumn{12}{|c|}{ Frequency of home cooking (n/week) } \\
\hline$\geq 5$ /week & 1310 & 14.3 & 23.3 & 5.8 & 1.2 & $<0.001$ & 8762 & 82.7 & 84.6 & 51.8 & $<0.001$ \\
\hline 3-4/week & 757 & 8.3 & 12.5 & 4.6 & 1.1 & & 902 & 8.5 & 8.3 & 11.8 & \\
\hline
\end{tabular}


Table 1 Characteristics of older Japanese men and women by level of cooking skills ( $n=19,378$ ) (Continued)

\begin{tabular}{|c|c|c|c|c|c|c|c|c|c|c|c|}
\hline & \multicolumn{6}{|c|}{ Males $(n=9143)$} & \multicolumn{5}{|c|}{ Females $(n=10,595)$} \\
\hline & \multicolumn{2}{|l|}{$\overline{\text { Total }}$} & \multicolumn{4}{|c|}{ Cooking skill } & \multicolumn{2}{|l|}{ Total } & \multicolumn{3}{|c|}{ Cooking skill } \\
\hline & $n$ & $\%$ & High & Middle & Low & $p$-value & $n$ & $\%$ & High & Middle/Low & $p$-value \\
\hline & & & $\%$ & $\%$ & $\%$ & & & & $\%$ & $\%$ & \\
\hline 1-2/week & 1002 & 11 & 15.3 & 7.7 & 2.0 & & 366 & 3.5 & 3.2 & 8.1 & \\
\hline$<1 /$ week & 1157 & 12.7 & 15.2 & 12.4 & 2.8 & & 199 & 1.9 & 1.5 & 7.2 & \\
\hline Never & 4917 & 53.8 & 33.7 & 69.6 & 92.9 & & 366 & 3.5 & 2.3 & 21.1 & \\
\hline \multicolumn{12}{|c|}{ Frequency of eating outside the home (n/week) } \\
\hline$\geq 5 /$ week & 242 & 2.6 & 2.6 & 2.3 & 3.7 & $<0.001$ & 103 & 1.0 & 0.9 & 1.4 & 0.001 \\
\hline 3-4/week & 462 & 5.1 & 5.0 & 5.4 & 4.2 & & 268 & 2.5 & 2.6 & 2.1 & \\
\hline 1-2/week & 1450 & 15.9 & 16.9 & 15.6 & 12.3 & & 1268 & 12.0 & 12.1 & 10.4 & \\
\hline$<1 /$ week & 3773 & 41.3 & 41.3 & 41.9 & 39.2 & & 4297 & 40.6 & 41.0 & 34.1 & \\
\hline Never & 3216 & 35.2 & 34.2 & 34.7 & 40.7 & & 4659 & 44.0 & 43.5 & 52.0 & \\
\hline \multicolumn{12}{|c|}{ Frequency of vegetable/fruit intake (n/day) } \\
\hline$\geq 1$ /day & 6625 & 72.5 & 73.3 & 70.6 & 74.4 & 0.008 & 9052 & 85.4 & 86.0 & 75.9 & $<0.001$ \\
\hline$<1$ /day (low frequency) & 2518 & 27.5 & 26.7 & 29.4 & 25.6 & & 1543 & 14.6 & 14.0 & 24.1 & \\
\hline \multicolumn{12}{|c|}{ Body weight status (BMI, kg/m²) } \\
\hline Underweight $(<18.5)$ & 428 & 4.7 & 4.1 & 5.1 & 5.8 & 0.04 & 988 & 9.3 & 9.2 & 11.8 & 0.08 \\
\hline Normal (18.5-27.4) & 8044 & 88.0 & 88.1 & 88.0 & 87.4 & & 8833 & 83.4 & 83.5 & 81.5 & \\
\hline Obesity ( $\geq 27.5$ ) & 671 & 7.3 & 7.8 & 6.9 & 6.9 & & 774 & 7.3 & 7.3 & 6.7 & \\
\hline
\end{tabular}

$B M I$ body mass index

dishes) [35]. The basic Japanese cooking methodsGohou (five methods) - are raw food, boiling, grilling, steaming, and frying [36]. We adopted stewing instead of steaming to reflect contemporary cooking practices [37]. Therefore, we included these elements and designed the following seven items for the Japanese version of the cooking skills scale: "How do you assess your overall cooking skills?"; "Can you peel fruits and vegetables?"; "Can you boil eggs and vegetables?"; "Can you grill fish?"; "Can you make stir-fried meat and vegetables?"; "Can you make miso soup?"; and "Can you make stewed dishes?" Participants were asked to evaluate their own cooking skills on a six-point scale (ranging from 1 for unable to 6 for very well). Cronbach's $\alpha$ for these seven items was 0.96 . Cronbach's $\alpha$ was calculated using an unstandardized approach for respondents answering five or more of the seven items. The mean of the seven items was calculated for each respondent to reflect their overall cooking skills; the midpoint was 3.5 , and a high score meant that the respondent had high confidence in their cooking skills (Table 2). The mean cooking skills score was divided into three categories-high (>4.0), middle $(2.1-4.0)$, and low $(\leq 2.0)$ - to examine the associations of cooking skills with body weight status and dietary behaviors. For women, because the distribution of the cooking skills score was skewed to the left (leaning towards higher scores), the middle and low groups were merged into one category. Therefore, women were classified into two cooking skills categories: high $(>4.0)$ and middle/low $(\leq 4.0)$.

\section{Person in charge of meal selection}

Participants were asked "In what way are your daily meals mainly prepared?" The responses to this item were as follows: cook by myself, a family member cooks, buy packaged lunches or cooked meals, use catering or homedelivery services, and other. Participants except for those who reported that a family member did the cooking were defined as being in charge of preparing or selecting meals.

\section{Covariates}

Covariates were assessed using the self-report questionnaire. Age was divided into four categories $(65-69,70$ $74,75-79$, and $\geq 80$ years). The duration of education was divided into three categories ( $\leq 9$ years, $10-12$ years, and $\geq 13$ years). Annual household income was adjusted for household size, dividing the household income by the square root of the number of people in the household. This variable was then divided into three categories $(<2.00,2.00-3.99$, and $\geq 4.00$ million yen). Marital status was divided into five categories (married, widowed, divorced, single, and other). To assess comorbidity, the participants were asked whether they were currently under medical treatment for any of the following conditions (multiple responses were allowed): cancer, heart 
disease, stroke, hypertension, diabetes mellitus, and hyperlipidemia. Covariates with missing data were categorized as "missing."

\section{Statistical analysis}

The analyses were stratified by gender because a previous study reported different associations between cooking skills and dietary behaviors by gender and distinct patterns of potential confounders for men and women [9]. First, participants were stratified by cooking skill level, and differences between groups were tested using Pearson's chi-squared tests. Second, multiple comparisons for the cooking skills scale were analyzed using the mixed linear model procedure to examine which cooking skills participants rated as difficult. The model adjusted for age, education, annual normalized household income, marital status, and medical treatment (cancer, heart disease, stroke, diabetes mellitus, hypertension, and hyperlipidemia), and peeling was used as the reference category. Participant identification code was included as a random effect. Third, we calculated adjusted odds ratios with 95\% confidence intervals (CIs) of high frequency of eating outside the home using logistic regression. For low frequency of home cooking and vegetable/fruit intake, we calculated adjusted prevalence ratios (APRs) with 95\% CIs using Poisson regression because participants with low frequencies of home cooking and vegetable/fruit intake were not uncommon, so the odds ratios derived from logistic regression would have been unable to approximate the prevalence ratio $[38,39]$. For the association with weight status, we calculated adjusted relative risk ratios (ARRRs) with 95\% CIs of underweight and obesity using multinomial logistic regression, with the body weight category of BMI of $18.5-27.4 \mathrm{~kg} / \mathrm{m}^{2}$ as the reference category. The models were adjusted for the following potential confounding factors: age, education, annual normalized household income, and medical treatment for cancer, heart disease, stroke, hypertension, diabetes mellitus, and hyperlipidemia. Participants with missing data on the covariates were included in the analysis. All analyses were conducted using Stata, Version 14 (Stata Statistical Software: Release 14. College Station, TX: StataCorp LP).

\section{Results}

The participants' characteristics are summarized in Table 1. A total of $46 \%$ of the participants were men, about $20 \%$ were aged over 80 years, 30\% had under 9 years of education, and $40 \%$ had annual incomes below two million yen. Of the male respondents, about $10 \%$ were widowed or divorced. When cognitive function was assessed with three items from the Kihon ChecklistCognitive Function scale, for which predictive validity for dementia incidence has previously been confirmed [40], only $0.9 \%$ of participants had three cognitive complaints. The majority of women (94.1\%) were classified as having a high level of cooking skills (Table 1). For men, the level of cooking skills was classified as high for $52.0 \%$, middle for $35.8 \%$, and low for $12.3 \%$. For women, $8.9 \%$ cooked less than two times a week, $3.5 \%$ ate out more than three times a week, $14.6 \%$ ate vegetables/ fruits less than once a day, 9.3\% were underweight, and $7.3 \%$ were obese. For men, $53.8 \%$ never cooked, $7.7 \%$ ate out more than three times a week, $27.5 \%$ ate vegetables/ fruits less than once a day, $4.7 \%$ were underweight, and $7.3 \%$ were obese. Women with middle/low-level cooking skills tended to be older, have a low level of education, have low income, not be married, and list a family member as the main meal preparer (Table 1). For men, in addition to being older, having a low level of education, and having a family member as the main meal preparer, men who were married tended to have a low level of cooking skills (Table 1 ).

The mean cooking skills score was higher for women (5.6 points) than for men (4.1 points) $(\mathrm{t}(19736)=-99.6$, $p<0.001$ ) (Table 2). For psychometric testing, one factor with an eigenvalue over 1 was found, and this accounted for $80.5 \%$ of the variance. All factor loadings were 0.8 or higher. Men rated stewing and stir-frying as more difficult than peeling. Although women had statistically significant differences between the assessed cooking skills, in terms of substantive significance, they rated all the methods assessed on the cooking skills scale as being of similar difficulty (Table 2).

There were gender differences in the associations of cooking skills with unhealthy dietary behaviors and body weight status (Table 3). Women with a middle/low level of cooking skills were 3.35 times (95\% CI: 2.87-3.92) more likely to have a lower frequency of home cooking and 1.61 (95\% CI: 1.36-1.91) times more likely to have a lower frequency of vegetable/fruit intake, compared with women with a high level of cooking skills. As for weight status, women with a middle/low level of cooking skills were 1.29 (95\% CI: 0.99-1.67) times more likely to be underweight, compared with women with a high level of cooking skills. For men, compared with those with a high level of cooking skills, men with a middle or low level of skill were 1.98 (95\% CI: 1.86-2.11) times more likely and 2.56 (95\% CI: $2.36-2.77)$ times more likely, respectively, to have a lower frequency of home cooking. Regarding eating outside the home, compared with men with a high level of cooking skills, men with a low level of cooking skills were 1.30 (95\% CI: 1.01-1.67) times more likely to have a higher frequency of eating outside the home. There was a significant association with a low frequency of vegetable/fruit intake only among men with a middle level of cooking skills (APR: 1.15, 95\% CI: 1.06-1.26). As for weight status, compared with men with a high level of cooking skills, men with middle or 
Table 2 Cooking skills among older Japanese men $(n=9143)$ and women $(n=10,595)$

\begin{tabular}{|c|c|c|c|c|c|c|c|c|}
\hline \multirow[t]{2}{*}{ Items } & \multicolumn{4}{|l|}{ Males } & \multicolumn{4}{|c|}{ Females } \\
\hline & mean & SD & Coef & $p$-value & mean & SD & Coef & $p$-value \\
\hline Overall cooking skills & 3.15 & 1.53 & -1.60 & $<0.001$ & 4.99 & 1.12 & -0.77 & $<0.001$ \\
\hline Able to peel fruits and vegetables & 4.75 & 1.44 & reference & & 5.76 & 0.74 & reference & \\
\hline Able to boil eggs and vegetables & 4.58 & 1.56 & -0.17 & $<0.001$ & 5.78 & 0.73 & 0.02 & 0.007 \\
\hline Able to grill fish & 4.24 & 1.72 & -0.50 & $<0.001$ & 5.70 & 0.86 & -0.06 & $<0.001$ \\
\hline Able to make stir-fried meat and vegetables & 4.04 & 1.76 & -0.70 & $<0.001$ & 5.72 & 0.82 & -0.04 & $<0.001$ \\
\hline Able to make miso soup & 4.16 & 1.77 & -0.59 & $<0.001$ & 5.74 & 0.80 & -0.02 & 0.001 \\
\hline Able to make stewed dishes & 3.43 & 1.81 & -1.32 & $<0.001$ & 5.72 & 0.82 & -0.05 & $<0.001$ \\
\hline Cooking skill scale & 4.05 & 1.42 & & & 5.63 & 0.74 & & \\
\hline
\end{tabular}

Multiple comparisons between cooking skills scale were analyzed using the mixed linear models procedure adjusted for age, education, annual normalized household income, marital status and medical treatment (cancer, heart disease, stroke, diabetes mellitus, hypertension and hyperlipidemia). Participant identification code was included as a random effect

low skill levels were 1.29 (95\% CI: 1.04-1.60) times more likely and 1.43 (95\% CI: 1.06-1.92) times more likely, respectively, to be underweight. There was no significant association between cooking skills and obesity for either men $(p=0.33)$ or women $(p=0.40)$. Using the cutoff point of $\mathrm{BMI} \geq 23.0 \mathrm{~kg} / \mathrm{m}^{2}$ as overweight, we found that a low level of cooking skills was not associated with an increased risk of overweight (Supplementary Table 1).

Next, we focused on men in charge of meals. Over $90 \%$ of women $(n=9618)$ but only $26 \%$ of men $(n=$ 2358) were in charge of daily meals. In contrast to men not in charge of preparing meals, most men in charge of meals rated their cooking skills as high (Supplementary Table 2). Men in charge of meals tended to have low levels of education and low income and to be unmarried (e.g., widowed or divorced) (Supplementary Table 2). When the associations with unhealthy dietary behaviors and weight status were examined for men in charge of meals, the effect size increased (Table 4). Compared with men with a high level of cooking skills, men with middle- or low-level cooking skills were 4.22 (95\% CI: $3.42-$ 5.21) times more likely and 7.85 (95\% CI: 6.04-10.21) times more likely, respectively, to have a lower frequency of home cooking (Table 4). Regarding eating outside the home, compared with men with a high level of cooking skills, men with a low level of cooking skills were 2.28 (95\% CI: 1.36-3.82) times more likely to have a higher frequency of eating outside the home. In relation to low frequency of vegetable/fruit intake, the APR for men with a middle level of cooking skills was 1.32 (95\% CI: 1.15-1.53). Furthermore, compared with men with a high level of cooking skills, men with middle or low skill levels were 1.59 (95\% CI: 1.04-2.45) times more likely and 2.79 (95\% CI: 1.45-5.36) times more likely, respectively, to be underweight.

Table 3 Adjusted prevalence/odds ratios/relative risk ratios of unhealthy eating behaviors, underweight, and obesity by level of cooking skills among older Japanese men $(n=9203)$ and women $(n=10,847)$

\begin{tabular}{|c|c|c|c|c|c|c|}
\hline & & $\begin{array}{l}\text { Low frequency of } \\
\text { home cooking }^{\mathrm{a}}\end{array}$ & $\begin{array}{l}\text { High frequency of eating } \\
\text { outside the home }\end{array}$ & $\begin{array}{l}\text { Low frequency of vegetable } \\
\text { and fruit intake }\end{array}$ & Underweight $^{d}$ & Obesity $^{e}$ \\
\hline & & APR $(95 \% \mathrm{Cl})$ & AOR $(95 \% \mathrm{Cl})$ & APR $(95 \% \mathrm{Cl})$ & ARRR $(95 \% \mathrm{Cl})$ & ARRR $(95 \% \mathrm{Cl})$ \\
\hline Men & & & & & & \\
\hline Cooking skill & High & ref & ref & ref & & ref \\
\hline & Middle & $1.98(1.86-2.11)$ & $1.15(0.97-1.36)$ & $1.15(1.06-1.26)$ & $1.29(1.04-1.60)$ & $0.89(0.75-1.06)$ \\
\hline & Low & $2.56(2.36-2.77)$ & $1.30(1.01-1.67)$ & $1.04(0.91-1.18)$ & $1.43(1.06-1.92)$ & $0.88(0.68-1.14)$ \\
\hline Women & & & & & & \\
\hline Cooking skill & High & ref & ref & ref & & ref \\
\hline & Middle-Low & $3.35(2.87-3.92)$ & $0.97(0.62-1.52)$ & $1.61(1.36-1.91)$ & $1.29(0.99-1.67)$ & $0.87(0.62-1.21)$ \\
\hline $\begin{array}{l}\text { AOR adjusted } \\
\text { aLow frequenc) } \\
\text { b }{ }^{b} \text { High frequenc } \\
\text { cLow frequenc) } \\
\text { d Underweight: } \\
\text { eObesity: BMI } \geq \\
\text { The models we } \\
\text { mellitus, hyper }\end{array}$ & $\begin{array}{l}\text { dds ratio, } A P R \text { ad } \\
\text { of home cookin } \\
\text { of eating outsid } \\
\text { of vegetable an } \\
3 \mathrm{Ml}<18.5 \mathrm{~kg} / \mathrm{m}^{2} \\
27.5 \mathrm{~kg} / \mathrm{m}^{2}\end{array}$ & $\begin{array}{l}\text { the home: frequenc } \\
\text { ruit intake: frequenc } \\
\text { education, annual } \\
\text { pidemia) }\end{array}$ & $\begin{array}{l}R R R \text { adjusted relative risk rat } \\
\text { king } \leq 2 \text { times/week for won } \\
\text { f eating outside the home } \geq \\
\text { f vegetable and fruit intake }\end{array}$ & $\begin{array}{l}\text { BMI body mass index, } \mathrm{Cl} \text { confid } \\
\mathrm{n} \text { and } 0 \text { times/week for men } \\
\text { imes/week } \\
\text { I/day }\end{array}$ & $x^{2}$ & troke, diabetes \\
\hline
\end{tabular}


Table 4 Adjusted prevalence ratios/relative risk ratios of low frequency of vegetable/fruit intake and home cooking, underweight, and obesity by cooking skills level among older Japanese men in charge of meals $(n=2370)$

\begin{tabular}{|c|c|c|c|c|c|c|}
\hline & & $\begin{array}{l}\text { Low frequency of } \\
\text { home cooking }^{\text {a }}\end{array}$ & $\begin{array}{l}\text { High frequency of } \\
\text { eating out }\end{array}$ & $\begin{array}{l}\text { Low frequency of vegetable } \\
\text { and fruit intake } e^{c}\end{array}$ & Underweight $^{d}$ & Obesity $^{\mathrm{e}}$ \\
\hline & & APR $(95 \% \mathrm{Cl})$ & AOR $(95 \% \mathrm{Cl})$ & APR $(95 \% \mathrm{Cl})$ & ARRR $(95 \% \mathrm{Cl})$ & ARRR $(95 \% \mathrm{Cl})$ \\
\hline \multicolumn{7}{|c|}{ Men in charge of cooking } \\
\hline \multirow[t]{3}{*}{ Cooking skill } & High & ref & & ref & ref & ref \\
\hline & Middle & $4.22(3.42-5.21)$ & $1.63(1.22-2.17)$ & $1.32(1.15-1.53)$ & $1.59(1.04-2.45)$ & $0.91(0.63-1.32)$ \\
\hline & Low & $7.85(6.04-10.21)$ & $2.28(1.36-3.82)$ & $1.12(0.84-1.50)$ & $2.79(1.45-5.36)$ & $0.59(0.25-1.39)$ \\
\hline
\end{tabular}

$A O R$ adjusted odds ratio, $A P R$ adjusted prevalence ratio, $A R R R$ adjusted relative risk ratio, $B M I$ body mass index, $C l$ confidence interval

a Low frequency of home cooking: frequency of home cooking 0 times/week

${ }^{b}$ High frequency of eating outside the home: frequency of eating outside the home $\geq 3$ times/week

'Low frequency of vegetable and fruit intake: frequency of vegetable and fruit intake $<1 /$ day

${ }^{\mathrm{d}}$ Underweight: $\mathrm{BMI}<18.5 \mathrm{~kg} / \mathrm{m}^{2}$

eObesity: $\mathrm{BMI} \geq 27.5 \mathrm{~kg} / \mathrm{m}^{2}$

The models were adjusted for age, education, annual normalized household income, marital status, and medical treatment (cancer, heart disease, stroke, diabetes mellitus, hypertension, and hyperlipidemia)

\section{Discussion}

To the best of our knowledge, this is the first study to examine the associations of cooking skills with unhealthy dietary behaviors and weight status by gender and meal preparer status among older adults. Using an adapted version of an existing cooking scale for use in Japanese populations, we confirmed that women had higher levels of cooking skills than did men and that the associations of cooking skills with dietary behaviors and weight status differed by gender. For both men and women, a low or middle/low level of cooking skills was associated with a low frequency of home cooking. Having low- or middle/low-level cooking skills was found to be significantly associated with high frequency of eating outside the home and with being underweight for men but not for women. The association between low or middle/low level of cooking skills and low frequency of vegetable/fruit intake was found for both men and women, but among men there was no dose-response relationship. The associations of low level of cooking skills with unhealthy dietary behaviors and underweight status were especially pronounced among men in charge of meals. Cooking skills were unassociated with obesity among both women and men.

In this study, a cooking skills scale for use in Japanese populations was designed with consideration of basic Japanese cooking methods and typical meals. Although we did not confirm the validity of this newly designed cooking skills scale by objective assessment, we were able to obtain plausible results, with the same trends observed with the original cooking skills scale for European populations [9]. Our cooking skills scale had appropriate internal consistency (Cronbach's $\alpha=0.96$ ) and showed higher values for women and those with higher education levels, which is consistent with previous findings $[9,41]$. This result also supports previous findings of a gender difference in confidence in cooking skills indicating that women are more confident in their cooking skills than are men [9, 22, 42]. The differences in cooking skills by gender and educational attainment among Japanese older adults may be explained by opportunities to learn cooking skills in school. In Japan, cooking education in schools was conducted exclusively for women until 1989; therefore, the men in this study (born before 1958) had less opportunity to learn cooking in school [43]. Another factor is that older age is associated with a stronger belief in the gender role ideology holding that men should work outside the home and women should do housework inside the home. In earlier years, there was even a cultural idea that men should not so much as enter the kitchen. This idea is reflected in the saying "Danshi-chubo-ni-hairazu" ("A man would be ashamed to be found in the kitchen") [44]. However, men's mean (SD) cooking skills score of 4.1 (1.42) was higher than the midpoint of 3.5, indicating that older Japanese men have above-average confidence in their cooking skills. In line with previous studies on adults showing that levels of cooking skills tend to be high in multiple-person households $[8,9]$, we found that women's cooking skills were higher when they were married than when they were not. However, in contrast, men's cooking skills were higher when they were not married (e.g., widowed or divorced). This result is intuitive because unmarried men do not have a spouse who is responsible for the cooking. We speculate that unmarried women have a moderate level of cooking skills because they were taught to cook in home economics classes and by their mothers, but their cooking skills may not have continued to improve because there was no need to cook for another person. Interventions earlier in the life course, such as at retirement, may be effective because men have a high risk of unhealthy eating behavior caused by their poor cooking skills if they are later widowed or divorced.

We included five basic cooking methods in the cooking skills scale, finding that men rated stewing and stirfrying as more difficult, compared with peeling and boiling. A previous study that examined eight cooking 
methods in the United Kingdom showed that men were more confident about boiling, compared with stewing or stir-frying [42]. This result is plausible because stewing and stir-frying require adjusting the level of heat and adding seasoning to prepare the dish properly. In interventions targeting men, it might be most beneficial to focus on simple cooking methods using stewing and stir-frying.

As expected, having a low or middle/low level of cooking skills was significantly associated with having a low frequency of home cooking for both men and women. People with high levels of cooking skills may enjoy cooking and feel self-confident regarding their cooking, leading to a high frequency of home cooking [9, 42]. However, a significant association between a low level of cooking skills and high frequency of eating outside the home was found only among men. This result may reflect the gender difference in the prevalence of eating outside the home. The percentage of respondents who ate out at least once a week was $15.5 \%$ for women and $23.6 \%$ for men (Table 2 ). A previous nationally representative survey in Japan reported that this gender difference exists across all age groups in the country, suggesting that men tend to prefer eating outside the home [45]. The same national survey also reported that the percentage of people who consume packaged lunches or cooked meals is the same for both men and women [45]. Therefore, women may consume these meals rather than eating outside the home, even if they have a low level of cooking skills. Another possible reason for the gender difference in eating outside the home is that there is a fundamental difference in cooking ability between men and women. In other words, the men who were categorized as having a low level of cooking skills cannot prepare any kind of meal, but the women who were categorized as having a middle/low level of cooking skills may be able to make basic meals. In our study, $79 \%$ of the women categorized as having a middle/low level of cooking skills were originally in the middle-level cooking skills category (Table 1). Therefore, women may not have to rely on eating outside the home even if they have a middle/low level of cooking skills.

Unlike home cooking and eating outside the home, no dose-response relationship between cooking skills and low vegetable/fruit intake was observed among men. In a previous study using the original cooking skills scale in Switzerland, favorable associations between cooking skills and various food groups including vegetables and fruits were evident for women, but these associations were weak or nonexistent for men [9]. This gender difference may be explained by nutritional knowledge [22, 46]: Men may not have sufficient nutritional knowledge regarding healthy food choice, even if they have a high level of cooking skills. Additionally, men may be more likely to choose foods because of their sensory appeal rather than for health reasons [47]. Future work should investigate food skills, including meal planning, food safety, and nutrition knowledge [23].

A low level of cooking skills was associated with underweight but not with obesity. Although a low level of cooking skills was associated with a high frequency of eating outside the home among men, which is generally associated with obesity, the majority of older Japanese people participating in this study did not rely on eating outside the home. The percentage of people who ate out more than three times a week was only $5 \%$ for the study participants, compared with 35\% for older Americans aged 60 years or older [29]. In our sample, more than $90 \%$ of the participants reported that their daily meals were mainly cooked by themselves or a family member (Table 1). Therefore, for older Japanese people, a low cooking frequency because of a low level of cooking skills may mean that they skip meals or eat simple meals or meals with poor nutritional value instead of eating outside the home. This would be more likely to lead to underweight than to obesity. A study examining the amount of rice served at local and chain restaurants in Japan found that most restaurants set the rice potion at an appropriate quantity for middle-aged and older people ( $>160 \mathrm{~g}$ and $<200 \mathrm{~g}$ ) [48]. Therefore, those eating outside the home in Japan may be unlikely to consume a high number of calories. When we additionally included frequency of home cooking and vegetable/fruit intake as potential mediators in our model, the ARRR of underweight decreased and became statistically non-significant among men, although it remained significant among men in charge of meals (Supplementary Table 3, Model 2). Another possible explanation for the association between cooking skills and underweight is that cooking skills may be a surrogate indicator of physical capacity in daily living. To examine this hypothesis, we included limitations in instrumental activities of daily living status $[49,50]$ in our model as a confounding factor, confirming that the ARRR of underweight remained significant (Supplementary Table 3, Model 3). Contrary to expectations, a low level of cooking skills was associated with underweight but not with obesity among older adults. For Asian people, underweight is a consistent risk factor for death, and this risk is higher than that associated with obesity [51]. Underweight has also been reported to be associated with frailty [52], fracture, and bone loss [53], which are critical obstacles to maintaining quality of life among older people [54, 55]. Therefore, it may be important for health policy makers to identify people with poor cooking skills and organize programs to enhance cooking skills to prevent underweight.

The association between cooking skills and underweight was especially prominent among men in charge 
of meals, and this association remained significant in this group even after accounting for limitations in instrumental activities of daily living, frequency of home cooking, and vegetable/fruit intake (Supplementary Table 3, Model 4). This finding is plausible because many men have family members-often their wives-who prepare meals for them. Among the men in our study sample, $74 \%$ reported that a family member prepared their daily meals (Table 1 ). Considering that many men in charge of meals are widowed or divorced (Supplementary Table 2), these men may have difficulty preparing meals because they had few opportunities to prepare meals before losing their spouse. These men may thus be less motivated to cook and to eat, which can lead to a lower appetite [56]. Additionally, appetite decreases with age, and poor appetite has been shown to be related to, for example, lower intake of energy, protein, and vegetables/fruits; lower dietary diversity [57, 58]; and higher risks of malnutrition [59] and mortality [58]. Further studies are needed to examine the potential mechanisms of the risk of underweight caused by low levels of cooking skills for men. About half of older Japanese men who cook at home started cooking when they were over 50 years old [60]. Considering that cooking classes for men have increased over the past few decades in Japan, intervening at an older age may be feasible and acceptable.

This study had several limitations. First, we used selfreported weight and height to calculate BMI, which may have led to an under- or over-estimate of BMI [61]. A previous study demonstrated that, when calculated from self-reported weight and height, the BMI of older Japanese people was underestimated, compared with objective measures of weight and height; however, the same study showed that the BMI of underweight men and women was overestimated by 0.7 and 0.3 , respectively, because these groups tended to over-report their weight [62]. Therefore, we may have underestimated the association of cooking skills with underweight. Second, we defined home cooking simply, excluding only the preparation of ready-to-eat food. Therefore, people who cooked low-quality meals or used some prepared foods in their cooking may have been included in the high frequency of home cooking category. This may have led to an underestimate of the association of cooking skills with low frequency of home cooking. Third, frequency of vegetable/fruit intake was assessed using a single, simple item. Future studies should use more detailed questions to assess which food groups are associated cooking skills. Fourth, we observed a ceiling effect for women's cooking skills, as has been reported in a previous study using the original cooking scale [9]. Considering the reduction in the available time for cooking in recent years, it may be beneficial to investigate cooking skills not only in terms of methods (e.g., boiling and stewing), but also in terms of the ability to prepare a variety of meals in a short time. Moreover, a thorough psychometric assessment of the modified cooking scale should be performed if it continues to be used. More comprehensive, validated measures for assessing food and cooking skill confidence are now available [63]. Therefore, it is possible to use these measurement methods in the future. Fifth, we could only evaluate a limited number of eating behaviors. Future work should examine associations between cooking skills and other aspects of diet, such as dietary pattern, food components, and portion size, to understand the mechanisms of the relationship between cooking skills and weight status. Sixth, because the municipalities that participated in the JAGES survey were not randomly selected, the generalizability of our findings to other populations in Japan is limited. Finally, because this study was cross-sectional, we could not assess causality: Reverse causation is possible, and unmeasured factors such as personality may confound the examined associations. For example, underweight may be accompanied by frailty or low muscle strength, which may make it difficult to cook some foods or to cook for a long time while standing, resulting in low confidence in one's cooking skills. However, more than half of the adult respondents in a previous study said that they had learned most of their cooking skills when they were teenagers and that they had learned these cooking skills mainly from their mothers [64]. Future randomized controlled trials comparing cooking, eating behaviors, and weight status among older adults with and without cooking skills interventions would clarify the causal association.

\section{Conclusions}

Using a large-scale cross-sectional study, we confirmed that women had higher levels of cooking skills than did men and that the associations of cooking skills with dietary behaviors and weight status differed by gender. Moreover, the associations of cooking skills with unhealthy dietary behaviors and weight status were especially pronounced among men who were in charge of meals. Considering the possibility that the person in charge of meals may change in older age, research on support to improve cooking skills among older people is needed.

\section{Supplementary information}

Supplementary information accompanies this paper at https://doi.org/10. 1186/s12966-020-00986-9.

Additional file 1: Table S1. Adjusted relative risk ratios of underweight and overweight according to the cooking skills of older Japanese men $(n=9,143)$ and women $(n=10,595)$. Table S2. Characteristics of older Japanese men by cooking responsibility status $(n=9,203)$. Table S3. 
Adjusted relative risk ratios of underweight by cooking skill among older Japanese men.

\section{Abbreviations}

Cl: confidence interval; BMI: body mass index; JAGES: Japan Gerontological Evaluation Study

\section{Acknowledgments}

We are particularly grateful to the staff members in each study area and in the central office for conducting the survey.

\section{Authors' contributions}

YT conceived the design, analyzed the data, reviewed the literature, and wrote the first draft of the article. KK collected the data. TF revised the first draft. KK edited the manuscript. All authors approved the final version of the manuscript.

\section{Funding}

This study used data from the JAGES, which was supported by a Japan Society for the Promotion of Science KAKENHI Grant (JP15H01972, 20H00557 and 19 K14029), a Health Labour Sciences Research Grant (H28-Choju-Ippan002, H30-Junkanki-Ippan-004, 19FA1012 and 19FA2001), grants from the Japan Agency for Medical Research and Development (JP18dk0110027, JP18ls0110002, JP18le0110009, JP19dk0110034 and JP20dk0110034), Research Funding for Longevity Sciences from National Center for Geriatrics and Gerontology (29-42), and a JST-OPERA program grant (JPMJOP1831).

\section{Availability of data and materials}

The datasets used and analyzed in the current study are from the JAGES study. All enquiries are to be addressed to the JAGES data management committee via e-mail: dataadmin.ml@jages.net. All JAGES datasets have ethical or legal restrictions for public deposition because of the inclusion of sensitive information from the human participants.

\section{Ethics approval and consent to participate}

The JAGES participants were informed that participation in the study was voluntary and that completing and returning the questionnaire via mail indicated their consent to participate in the study. The JAGES protocol was approved by the Ethics Committee in Research of Human Subjects at the National Center for Geriatrics and Gerontology (No. 992) and Chiba University Faculty of Medicine (No. 2493)

\section{Consent for publication}

Not applicable.

\section{Competing interests}

The authors declare that they have no competing interests.

\section{Author details}

${ }^{1}$ Department of Global Health Promotion, Tokyo Medical and Dental University (TMDU), 1-5-45, Yushima, Bunkyo-ku, Tokyo 113-8519, Japan. ${ }^{2}$ Center for Preventive Medical Sciences, Chiba University, 1-8-1 Inohana, Chuo-ku, Chiba-shi, Chiba 260-8672, Japan. ${ }^{3}$ Department of Gerontological Evaluation, Center for Gerontology and Social Scienc, National Center for Geriatrics and Gerontology, 7-430 Morikoka-cho, Obu-shi, Aichi 474-8511, Japan

Received: 20 November 2019 Accepted: 18 June 2020 Published online: 26 June 2020

\section{References}

1. Lichtenstein AH, Ludwig DS. Bring back home economics education. Jama. 2010;303:1857-8.

2. Mills $\mathrm{S}$, White $\mathrm{M}$, Brown $\mathrm{H}$, Wrieden W, Kwasnicka D, Halligan J, et al. Health and social determinants and outcomes of home cooking: a systematic review of observational studies. Appetite. 2017;111:116-34.

3. Tiwari A, Aggarwal A, Tang W, Drewnowski A. Cooking at home: a strategy to comply with U.S. dietary guidelines at no extra cost. Am J Prev Med. 2017:52:616-24.
4. Mills S, Brown H, Wrieden W, White M, Adams J. Frequency of eating home cooked meals and potential benefits for diet and health: cross-sectional analysis of a population-based cohort study. Int J Behav Nutr Phys Act. 2017:14:109.

5. Chen RC, Lee MS, Chang YH, Wahlqvist ML. Cooking frequency may enhance survival in Taiwanese elderly. Public Health Nutr. 2012;15:1142-9.

6. Smith LP, Ng SW, Popkin BM. Trends in US home food preparation and consumption: analysis of national nutrition surveys and time use studies from 1965-1966 to 2007-2008. Nutr J. 2013;12:45.

7. Statistics Bureau, Ministry of Internal Affairs and Communications. Family income and expenditure survey FY2015; 2019. http://www.stat.go.jp/data/ kakei/longtime/. Published 2016. Accessed 29 August. [in Japanese].

8. van der Horst K, Brunner TA, Siegrist M. Ready-meal consumption: associations with weight status and cooking skills. Public Health Nutr. 2011; 14:239-45.

9. Hartmann C, Dohle S, Siegrist M. Importance of cooking skills for balanced food choices. Appetite. 2013;65:125-31.

10. Lam MCL, Adams J. Association between home food preparation skills and behaviour, and consumption of ultra-processed foods: cross-sectional analysis of the UK National Diet and nutrition survey (2008-2009). Int J Behav Nutr Phys Act. 2017;14:68.

11. Flego A, Herbert J, Waters E, Gibbs L, Swinburn B, Reynolds J, et al. Jamie's Ministry of Food: quasi-experimental evaluation of immediate and sustained impacts of a cooking skills program in Australia. PLoS One. 2014;9:e114673.

12. Reicks $M$, Kocher M, Reeder J. Impact of cooking and home food preparation interventions among adults: a systematic review (2011-2016). J Nutr Educ Behav. 2018:50:148-72.e1.

13. World Health Organization. Ageing and health: World Health Organization; 2018. https://apps.who.int/iris/handle/10665/279010. Accessed 23 Jun 2018.

14. World Health Organization. Keep fit for life: meeting the nutritional needs of older persons. Geneva: World Health Organization; 2002.

15. Robinson SM. Improving nutrition to support healthy ageing: what are the opportunities for intervention? Proc Nutr Soc. 2018;77:257-64.

16. Dean $M$, Raats $M M$, Grunert KG, Lumbers M. Factors influencing eating a varied diet in old age. Public Health Nutr. 2009;12:2421-7.

17. Porter Starr K, McDonald SD, Bales C. Nutritional vulnerability in older adults: a continuum of concerns. Curr Nutr Rep. 2015;4:176-84.

18. Conklin Al, Maguire ER, Monsivais P. Economic determinants of diet in older adults: systematic review. J Epidemiol Community Health. 2013;67:721-7.

19. Hasan B, Thompson WG, Almasri J, Wang Z, Lakis S, Prokop L, et al. The effect of culinary interventions (cooking classes) on dietary intake and behavioral change: a systematic review and evidence map. BMC Nutr. 2019; 5:29.

20. Bongaarts J, Zimmer Z. Living arrangements of older adults in the developing world: an analysis of demographic and health survey household surveys. J Gerontol B Psychol Sci Soc Sci. 2002;57:S145-57.

21. National Institute of Population and Social Security Research. Household projections for Japan 2010-2035: National Institute of Population and Social Security Research; 2013. http://www.ipss.go.jp/ppajsetai/e/hhpri2013/tpage_e.asp. Accessed 1 Jun 2017. [in Japanese].

22. Caraher M, Dixon P, Lang T, Carr R. The state of cooking in England: the relationship of cooking skills to food choice. Br Food J. 1999;101:590-609.

23. McGowan L, Caraher M, Raats M, Lavelle F, Hollywood L, McDowell D, et al. Domestic cooking and food skills: a review. Crit Rev Food Sci Nutr. 2017;57: 2412-31.

24. Kondo K. Progress in aging epidemiology in Japan: the JAGES project. J Epidemiol. 2016;26:331-6.

25. Kondo K, Rosenberg M, Organization WH. Advancing universal health coverage through knowledge translation for healthy ageing: lessons learnt from the Japan Gerontological evaluation study. Geneva: World Health Organization; 2018.

26. Tamiya N, Noguchi H, Nishi A, Reich MR, lkegami N, Hashimoto H, et al. Population ageing and wellbeing: lessons from Japan's long-term care insurance policy. Lancet (London, England). 2011;378:1183-92.

27. Katz S, Downs TD, Cash HR, Grotz RC. Progress in development of the index of ADL. Gerontologist. 1970;10:20-30

28. WHO Expert Consultation. Appropriate body-mass index for Asian populations and its implications for policy and intervention strategies. Lancet. 2004:363:157-63. 
29. Kant AK, Whitley MI, Graubard BI. Away from home meals: associations with biomarkers of chronic disease and dietary intake in American adults, NHANES 2005-2010. Int J Obes. 2015;39:820-7.

30. Tani Y, Kondo N, Takagi D, Saito M, Hikichi H, Ojima T, et al. Combined effects of eating alone and living alone on unhealthy dietary behaviors, obesity and underweight in older Japanese adults: results of the JAGES. Appetite. 2015;95:1-8.

31. Yanagi N, Hata A, Kondo K, Fujiwara T. Association between childhood socioeconomic status and fruit and vegetable intake among older Japanese: the JAGES 2010 study. Prev Med. 2018;106:130-6.

32. Okuda N, Miura K, Okayama A, Okamura T, Abbott RD, Nishi N, et al. Fruit and vegetable intake and mortality from cardiovascular disease in Japan: a 24-year follow-up of the NIPPON DATA 80 study. Eur J Clin Nutr. 2015;69:482-8.

33. Nagura J, Iso H, Watanabe Y, Maruyama K, Date C, Toyoshima H, et al. Fruit, vegetable and bean intake and mortality from cardiovascular disease among Japanese men and women: the JACC study. Br J Nutr. 2009;102: 285-92.

34. Takachi R, Inoue M, Ishihara J, Kurahashi N, Iwasaki M, Sasazuki S, et al. Fruit and vegetable intake and risk of total cancer and cardiovascular disease: Japan public health center-based prospective study. Am J Epidemiol. 2008; 167:59-70.

35. Ministry of Agriculture, Forestry and Fisheries. WASHOKU [Available from: http://www.maff.go.jp/j/keikaku/syokubunka/culture/pdf/guide_all.pdf. Accessed 6 February, 2018. [in Japanese].

36. Higashi A. Washoku and health. Chem Educ. 2015;63:38-9.

37. Nakatani K, Masui H. Survey on awareness of steam cooking and actual cooking. In: The 61 st Japan Society of Home Economics Hyogo: the Japan Society of Home Economics; 2009. in Japanese.

38. Zhang J, Yu KF. What's the relative risk? A method of correcting the odds ratio in cohort studies of common outcomes. Jama. 1998;280:1690-1.

39. Barros AJ, Hirakata VN. Alternatives for logistic regression in cross-sectional studies: an empirical comparison of models that directly estimate the prevalence ratio. BMC Med Res Methodol. 2003;3:21.

40. Tomata Y, Sugiyama K, Kaiho Y, Sugawara Y, Hozawa A, Tsuji I. Predictive ability of a simple subjective memory complaints scale for incident dementia: evaluation of Japan's national checklist, the "Kihon checklist" Geriatr Gerontol Int. 2017;17:1300-5.

41. McGowan L, Pot GK, Stephen AM, Lavelle F, Spence M, Raats M, et al. The influence of socio-demographic, psychological and knowledge-related variables alongside perceived cooking and food skills abilities in the prediction of diet quality in adults: a nationally representative crosssectional study. Int J Behav Nutr Phys Act. 2016;13:111.

42. Adams J, Goffe L, Adamson AJ, Halligan J, O'Brien N, Purves R, et al. Prevalence and socio-demographic correlates of cooking skills in UK adults: cross-sectional analysis of data from the UK National Diet and nutrition survey. Int J Behav Nutr Phys Act. 2015;12:99.

43. Nishikawa $Y$, Ohuchi $H$, Suzuki M, Ohmura N. The future of cooking practice in home economics. Bull Coll Educ Ibaraki Univ (Educational Sciences). 2008; 57:117-28 in Japanese.

44. Iwai N. Husband's participation in housework among elderly couples -effects of work status, health condition, caring experience, household structure and gender role ideology of wives and husbands. Tokyo: The national survey of Social Stratification and social Mobility; 1995. [in Japanese].

45. Ministry of Health, Labour and Welfare. National Health and nutrition survey FY2015. Tokyo: Ministry of Health, Labour and Welfare; 2015. https://www. mhlw.go.jp/stf/houdou/0000142359.html. Accessed 29 October 2019.[in Japanese].

46. Baker $\mathrm{AH}$, Wardle J. Sex differences in fruit and vegetable intake in older adults. Appetite. 2003;40:269-75.

47. Steptoe A, Pollard TM, Wardle J. Development of a measure of the motives underlying the selection of food: the food choice questionnaire. Appetite. 1995:25:267-84.

48. Shimpo M, Akamatsu R, Saiki M, Fujisaki K. Portion sizes of Rice and dishes at restaurants: a measurement survey of restaurants in the Tokyo, Japan, metropolitan area and an analysis using public information on chain restaurants. Jpn J Nutr Diet. 2019;77:105-12 [in Japanese]

49. Koyano W, Shibata H, Nakazato K, Haga H, Suyama Y. Measurement of competence: reliability and validity of the TMIG index of competence. Arch Gerontol Geriatr. 1991:13:103-16.
50. Tani Y, Suzuki N, Fujiwara T, Hanazato M, Kondo K. Neighborhood food environment and dementia incidence: the Japan Gerontological evaluation study cohort survey. Am J Prev Med. 2019;56:383-92.

51. Zheng W, McLerran DF, Rolland B, Zhang X, Inoue M, Matsuo K, et al. Association between body-mass index and risk of death in more than 1 million Asians. N Engl J Med. 2011;364:719-29.

52. Hubbard RE, Lang IA, Llewellyn DJ, Rockwood K. Frailty, body mass index, and abdominal obesity in older people. J Gerontol A Biol Sci Med Sci. 2010; 65:377-81.

53. Tanaka S, Kuroda T, Saito M, Shiraki M. Overweight/obesity and underweight are both risk factors for osteoporotic fractures at different sites in Japanese postmenopausal women. Osteoporos Int. 2013;24:69-76.

54. Kojima G, lliffe S, Jivraj S, Walters K. Association between frailty and quality of life among community-dwelling older people: a systematic review and meta-analysis. J Epidemiol Community Health. 2016;70:716-21.

55. Zanker J, Duque G. Osteoporosis in older persons: old and new players. J Am Geriatr Soc. 2019;67:831-40.

56. Pilgrim AL, Robinson SM, Sayer AA, Roberts HC. An overview of appetite decline in older people. Nurs Older People. 2015;27:29-35.

57. van der Meij BS, Wijnhoven HAH, Lee JS, Houston DK, Hue T, Harris TB, et al. Poor appetite and dietary intake in community-dwelling older adults. J Am Geriatr Soc. 2017:65:2190-7.

58. Huang YC, Wahlqvist ML, Lee MS. Appetite predicts mortality in free-living older adults in association with dietary diversity. A NAHSIT cohort study. Appetite. 2014;83:89-96.

59. O'Keeffe M, Kelly M, O'Herlihy E, O'Toole PW, Kearney PM, Timmons S, et al. Potentially modifiable determinants of malnutrition in older adults: a systematic review. Clin Nutr. 2018;38(6):2477.

60. Japan Frozen Food Association. Survey of dietary consciousness and actual conditions of senior generations targeting 1,000 men and women in their 60s. Tokyo; 2011. https://www.reishokukyo.or.jp/wp-content/uploads/ pdf/111020_news.pdf. Accessed 29 February, 2020. [in Japanese].

61. Connor Gorber S, Tremblay M, Moher D, Gorber B. A comparison of direct vs. self-report measures for assessing height, weight and body mass index: a systematic review. Obes Rev. 2007:8:307-26.

62. Ikeda N. Validity of self-reports of height and weight among the general adult population in Japan: Findings from National Household Surveys, 1986. PLoS One. 2016;11:e0148297.

63. Lavelle F, McGowan L, Hollywood L, Surgenor D, McCloat A, Mooney E, et al. The development and validation of measures to assess cooking skills and food skills. Int J Behav Nutr Phys Act. 2017;14:118.

64. Lavelle F, Spence M, Hollywood L, McGowan L, Surgenor D, McCloat A, et al. Learning cooking skills at different ages: a cross-sectional study. Int J Behav Nutr Phys Act. 2016:13:119.

\section{Publisher's Note}

Springer Nature remains neutral with regard to jurisdictional claims in published maps and institutional affiliations.

\section{Ready to submit your research? Choose BMC and benefit from:}

- fast, convenient online submission

- thorough peer review by experienced researchers in your field

- rapid publication on acceptance

- support for research data, including large and complex data types

- gold Open Access which fosters wider collaboration and increased citations

- maximum visibility for your research: over $100 \mathrm{M}$ website views per year

At $\mathrm{BMC}$, research is always in progress.

Learn more biomedcentral.com/submission 